\title{
Inhibitory roles for SHP-1 and SOCS-3 following pituitary proopiomelanocortin induction by leukemia inhibitory factor
}

\author{
Corinne Bousquet, ${ }^{1}$ Christiane Susini, ${ }^{2}$ and Shlomo Melmed ${ }^{1}$ \\ ${ }^{1}$ Department of Medicine, Cedars-Sinai Research Institute-University of California-Los Angeles School of Medicine, \\ Los Angeles, California 90048, USA \\ ${ }^{2}$ Institut National de la Santé et de la Recherche Médicale (INSERM) U151, Institut Louis Bugnard,
} 31054 Toulouse, France

Address correspondence to: Shlomo Melmed, Room 2015, Cedars-Sinai Medical Center, 8700 Beverly Boulevard, Los Angeles, California 90048, USA.

Phone: (310) 423-4691; Fax: (310) 423-0119; E-mail: Melmed@CSMC.edu.

Received for publication July 21, 1999, and accepted in revised form September 22, 1999.

Leukemia inhibitory factor (LIF) is a pleiotropic cytokine that stimulates the hypothalamo-pituitary-adrenal (HPA) axis through JAK-STAT activation. We show here that LIF-induced JAK2 and STAT3 tyrosine phosphorylation is transient, disappearing within 20 and 40 minutes, respectively. LIF activates the SH2 domain-containing tyrosine phosphatase, SHP-1, with maximal stimulation observed at 30 minutes. SHP-1 is constitutively associated with JAK2, and LIF induces recruitment of phosphorylated STAT3 to this complex. Overexpression of wild-type or dominant negative forms of SHP-1 shows decreased or increased LIF-induced proopiomelanocortin (POMC) promoter activity, respectively. LIF-induced JAK2 and STAT3 dephosphorylation is delayed until after 60 minutes in cells that overexpress the mutant SHP-1. In addition, SOCS-3, a negative regulator of LIF signaling, binds to JAK2 after 60 minutes of LIF stimulation, after which the complex is degraded by the proteasome. SOCS-3 overexpression blocks LIF-induced JAK2 tyrosine phosphorylation, confirming a role for SOCS-3 in deactivating JAK2 by direct association. Using SOCS-3 fusion proteins, we also define regions of the SOCS-3 protein that are critical for inhibition of LIF-induced POMC promoter activity. Corticotrophic signaling by LIF is thus subject to 2 forms of negative autoregulation: dephosphorylation of JAK2 and STAT3 by the SHP-1 tyrosine phosphatase, and SOCS3 -dependent inactivation of JAK2.

J. Clin. Invest. 104:1277-1285 (1999).

\section{Introduction}

Leukemia inhibitory factor (LIF) is a member of the IL-6 cytokine family (1). We have shown that LIF plays an important role in the pituitary by modulating the hypothalamo-pituitary-adrenal (HPA) axis response to stress or inflammation (2). Indeed, LIF receptor expression has been demonstrated in murine hypothalamus and pituitary and in murine corticotroph AtT20 cells. Human fetal and adult pituitary tissues also express the LIF receptor gene $(3,4)$. In the murine pituitary, in addition to corticotropin-releasing hormone $(\mathrm{CRH})$, LIF and LIF receptor gene expression are induced by stress and participate in upregulation of proopiomelanocortin (POMC) gene expression and adrenocorticotropin hormone (ACTH) secretion (2). In AtT20 cells, LIF also induces POMC transcription and ACTH secretion, and it synergizes with hypothalamic CRH to trigger these effects (5). CRH is a critical factor in the activation of the HPA axis, and the observations that cytokines such as LIF strongly impact this axis have established the concept of a neuroimmunoendocrine interface occurring at the level of the hypothalamic pituitary unit. Molecular mechanisms involved in cytokine signaling include ligand activation of a receptor complex, comprising a low-affinity signal-transducing receptor subunit (LIF receptor $\alpha$ subunit for LIF) and the signal-transducing gp130 subunit (1). Ligand binding is associated with the formation of a ligand-high affinity complex, which does not possess intrinsic kinase activity. Janus kinase (JAK) is, however, constitutively associated with gp130 (6). After ligand-induced receptor dimerization, JAK is responsible for tyrosine phosphorylation of gp130 sites. These sites also dock with SH2-containing proteins, such as STAT, and activate either PI3K or SHP-2 (1). After tyrosine phosphorylation, STAT homo- or heterodimerizes and translocates to the nucleus, where it transactivates several genes (7). We recently showed that by blocking STAT3 function in AtT20 cells, LIF-induced activation of ACTH secretion is strikingly abrogated (8). The cytokine-induced JAK-STAT phosphorylation pathway is transient, implying the presence of a plastic intracellular negative regulatory mechanism. Therefore, we tested molecular mechanisms that might downregulate the corticotroph LIF signaling pathway. 
SHP-1 is a cytoplasmic protein tyrosine phosphatase containing 2 Src homology 2 (SH2) domains (9). The phosphatase activity of SHP-1 positively regulates EGF- or IFN- $\gamma$-induced STAT activation in HeLa cells (10), but it is usually reported as a negative regulator (11-14). The negative effects of SHP-1 on cytokine receptor-mediated signaling occur by dephosphorylation of the cytokine receptor itself or receptor-associated phosphorylated mediators, such as JAK-2, and termination of JAK2-mediated signaling (11-14).

A new family of cytokine-induced immediate-early genes has recently been identified and includes 8 members, including cytokine-inducible $\mathrm{SH} 2$ protein (CIS) and suppressor of cytokine signaling 1-7 (SOCS-1-7) (15). A feature of these cytokine-inducible proteins is the negative regulation of cytokine transduction pathways $(16,17)$. We recently demonstrated that LIF induces pituicyte SOCS-3 gene expression in vitro and in vivo (18). Overexpression of SOCS-3 in AtT20 cells abrogated LIF-induced gp130 and STAT3 phosphorylation, POMC gene expression, POMC promoter activity, and ACTH secretion (18). Furthermore, LIF-induced SOCS-3 gene expression was partially STAT3 dependent, and SOCS-3 exhibits a negative autoregulatory feedback on its own gene expression, thus limiting SOCS-3 accumulation in the corticotroph cell (19).

We now investigate whether SHP-1 and/or SOCS-3 are components of the early negative feedback for LIFinduced JAK-STAT tyrosine phosphorylation in AtT20 cells, and we demonstrate that both SHP-1 and SOCS3 downregulate LIF signaling by using 2 distinct, but redundant, mechanisms for terminating pituitary LIF signaling to POMC.

\section{Methods}

Culture and transformation of AtT20 cells. AtT20 cells were grown in DMEM supplemented with 10\% FBS, 2 mM L-glutamine, $100 \mathrm{U} / \mathrm{mL}$ streptomycin, $100 \mathrm{U} / \mathrm{mL}$ penicillin, and $0.25 \mu \mathrm{g} / \mathrm{mL}$ amphotericin B (Life Technologies Inc., Gaithersburg, Maryland, USA). To isolate stable transformants expressing wild-type or mutated SHP-1, corresponding cDNAs were transfected in AtT20 cells by calcium precipitation and transformants were selected with G418 (1 mg/mL).

Immunoprecipitation and Western blotting analysis. AtT20 cells were grown for 48 hours and serum deprived for 16 hours before treatment with 1 nM LIF (R\&D Systems Inc., Minneapolis, Minnesota, USA). Cells lysis, protein immunoprecipitations, and Western blottings were performed as described elsewhere (8). For immunoprecipitations and Western blottings, monoclonal antiphosphatase SHP-1 (Transduction Laboratories, Lexington, Kentucky, USA) and antiphosphotyrosine (Santa Cruz Biotechnology Inc., Santa Cruz, California, USA) antibodies, or polyclonal anti-JAK2, anti-STAT3, and anti-SOCS-3 antibodies (Santa Cruz Biotechnology), prebound to Sepharose-protein G or A beads (Sigma Chemical Co., St. Louis, Missouri, USA), were used.
Tyrosine phosphatase assay. Cells were plated for 48 hours and serum deprived 16 hours before treatment with $1 \mathrm{nM}$ LIF. Tyrosine phosphatase activity was then assessed as described elsewhere (20). For each point, the SHP-1 activity was calculated after subtraction of the amount of phosphatase activity obtained with a nonimmune serum immunoprecipitate. Furthermore, SHP-1 phosphatase activity was normalized for the amount of total SHP-1 immunoprecipitated for each time point: one half of each immunoprecipitate was assessed for SHP-1 activity, and the other half was used to perform an anti-SHP-1 Western blot.

Construction of SOCS-3 fusion proteins. Defined segments of SOCS-3 were cloned by PCR using sense and antisense primers containing a BamHI or a XhoI site, respectively, and introduced in the $\mathrm{BamHI}$ /XhoI sites of pcDNA3.1/His vector (Invitrogen Corp., San Diego, California, USA). The fusion proteins containing the $\mathrm{X}$ press tag at their $5^{\prime}$ end were subcloned in the SmaI site of the PSVL vector (Pharmacia Biotech Inc., Piscataway, New Jersey, USA). The sequences of all constructs were verified by sequencing.

Transfection and luciferase assay. AtT20 cells were plated in 2-mL dishes (100,000 cells per well) and allowed to adhere for 16 hours. Cells were transiently transfected as described elsewhere (21) with $0.5 \mu \mathrm{g}$ per dish of rat POMC promoter $(-706 /+64)$ fused to the luciferase reporter gene and $0.5 \mu \mathrm{g}$ of the pSV-lacZ (Promega Corp., Madison, Wisconsin, USA) expressing $\beta$-galactosidase as an internal transfection control. In cotransfection experiments using SOCS-3 fusion proteins, 0.5 $\mu \mathrm{g}$ of each construct was cotransfected with the ratPOMC promoter construct and with the pSV-lacZ vector. Twenty-four hours after transfection, cells were treated with $1 \mathrm{nM}$ LIF in triplicate for 6 hours. Cells were then washed once in $\mathrm{PBS}$ ( $\mathrm{pH} 7.0)$, lysed in reporter

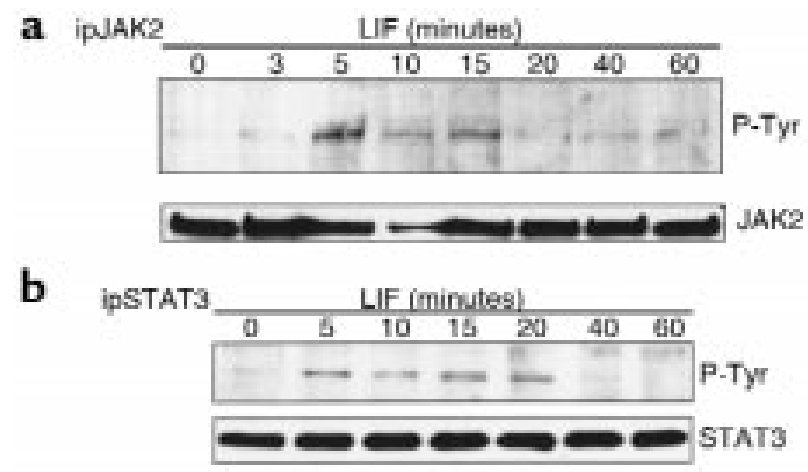

Figure 1

Time course of LIF-induced JAK2 and STAT3 phosphorylation. AtT20 cells were treated for the indicated times with $1 \mathrm{nM}$ LIF. After cell lysis and immunoprecipitation with polyclonal antibody recognizing either JAK2 (a) or STAT3 (b), immunoprecipitates were separated on a $7.5 \%$ polyacrylamide-SDS gel. Immunoblotting was then performed with a monoclonal antiphosphotyrosine antibody. To verify equal protein loading in each lane, membranes were stripped and immunoblotted with antibodies recognizing either JAK2 (a) or STAT3 (b). 
a

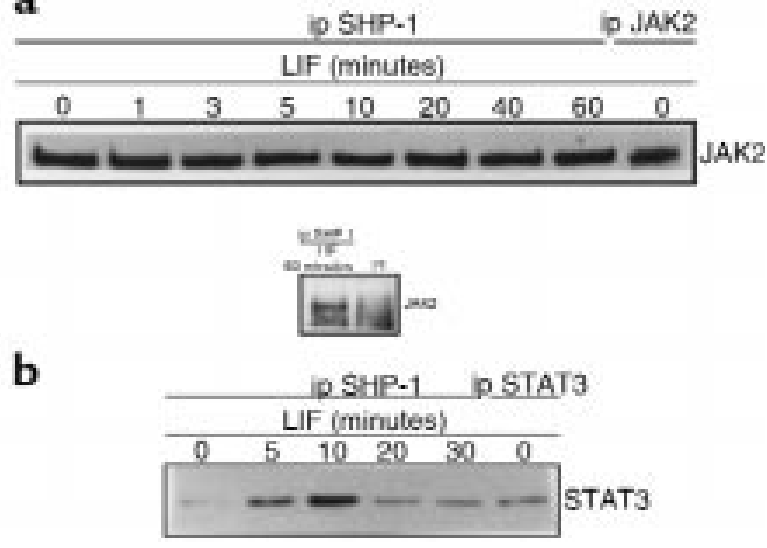

Figure 2

SHP-1 forms a constitutive complex with JAK2, and the recruitment of STAT3 is LIF-inducible. AtT20 cells were treated for the indicated times with $1 \mathrm{nM}$ LIF. After cell lysis, $500 \mu \mathrm{g}$ of protein extracts was immunoprecipitated with an $\mathrm{mAb}$ recognizing SHP-1 (a, lanes $1-8 ; \mathbf{b}$, lanes $1-5)$ or with a mouse preimmune serum (PI). To verify the identity of JAK2 and STAT3, $50 \mu \mathrm{g}$ of protein extracts was immunoprecipitated with polyclonal antibodies recognizing JAK2 (a, lane 9) and STAT3 (b, lane 6). Immunoprecipitates were separated on a $7.5 \%$ polyacrylamideSDS gel, and immunoblotting was performed with a polyclonal antiJAK2 antibody (a) or a polyclonal anti-STAT3 antibody (b).

lysis buffer, and subjected to assay for luciferase (21) or $\beta$-galactosidase activity (Promega Corp.).

\section{Results}

Time course of LIF-induced JAK2 and STAT3 phosphorylation. LIF triggers its effects by inducing the JAK-STAT transduction pathway, with JAK2 and STAT3 reported to be the principal proteins activated by LIF signaling (8). Therefore, we performed Western blotting analysis to analyze the kinetics of LIF-induced JAK2 and STAT3 phosphorylation (Figure 1). Proteins from solubilized cells were extracted at different time points after LIF induction. JAK2 (Figure 1a) and STAT3 (Figure 1b) phosphorylations were analyzed after immunoprecipitation of the solubilized proteins by using their respective antibodies (anti-JAK2 and anti-STAT3) and by Western blotting of the immunoprecipitated proteins with an antiphosphotyrosine antibody or anti-JAK2 and anti-STAT3 antibodies, respectively. JAK2 phosphorylation occurred as early as 5 minutes after LIF stimulation and disappeared within 20 minutes. The time course of STAT3 phosphorylation, a JAK2 substrate, was similar, but its dephosphorylation was slightly delayed: LIF-induced STAT3 phosphorylation was evident as early as 5 minutes and disappeared within 40 minutes. Thus, LIF-induced phosphorylations of JAK2 and STAT3 are transient, and a negative feedback mechanism might be responsible for these respective dephosphorylation reactions.

SHP-1 is constitutively bound to JAK2. Next, we investigated mechanisms accounting for the early dephosphorylation of LIF-signaling substrates. We tested whether the catalytic activity of SHP-1 tyrosine phos- phatase might be responsible for JAK2 substrate dephosphorylation in LIF signaling. We performed protein association studies using solubilized protein from LIF-treated AtT20 cells and antibodies recognizing SHP-1, JAK2, and STAT3. As shown in Figure 2a, JAK2 was detected within anti-SHP-1 immunoprecipitates, whereas no band was observed in an anti-mouse preimmune serum immunoprecipitate. In a cross-experiment, the presence of SHP-1 was confirmed within anti-JAK2 immunoprecipitates (data not shown). The appropriate size of the observed JAK2 band was verified by using anti-JAK2 immunoprecipitate in an anti-JAK2 Western blot (Figure 2a, lane 9). Equal protein loading was demonstrated by stripping and reblotting the membrane with an anti-SHP-1 antibody (data not shown). The association of SHP- 1 and JAK2 remained constant after LIF treatment. These results demonstrated that SHP-1 constitutively associates with JAK2 and that JAK2 might be a potential substrate for SHP-1.

Association between SHP-1 and STAT3 is LIF regulated. Next, we investigated whether STAT3 was a potential substrate for SHP-1. We performed a Western blotting analysis using an anti-STAT3 antibody to detect STAT3 in anti-SHP-1 immunoprecipitates. We observed an 89-kDa band corresponding in size to STAT3 (Figure $2 b$, lanes 1-5), which was confirmed by using an antiSTAT3 immunoprecipitate (Figure 2b, lane 6). The abundance of STAT3 coimmunoprecipitated with

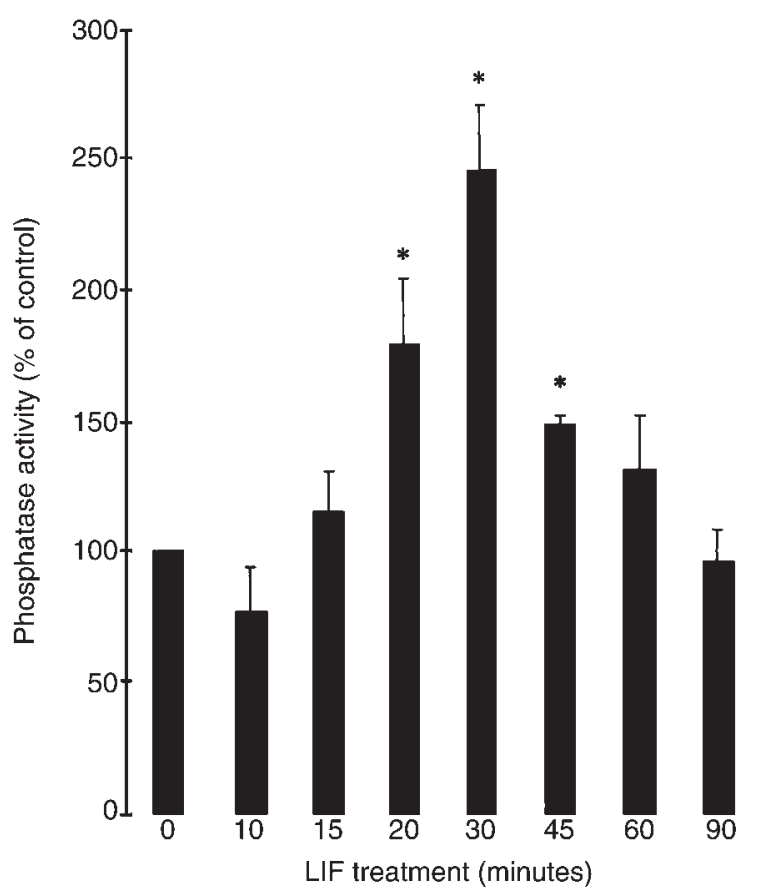

Figure 3

SHP-1 catalytic activity is LIF-inducible. AtT20 cells were stimulated for the indicated times with $1 \mathrm{nM}$ LIF. After cell lysis and immunoprecipitation with $\mathrm{mAb}$ recognizing SHP-1, immunoprecipitates were subjected to a phosphatase assay using [ $\left.{ }^{32} \mathrm{P}\right]$ poly (Glu, Tyr). The results are representative of 3 separate experiments and are expressed as percentage of control $(100 \%=$ no treatment $)$. 
a

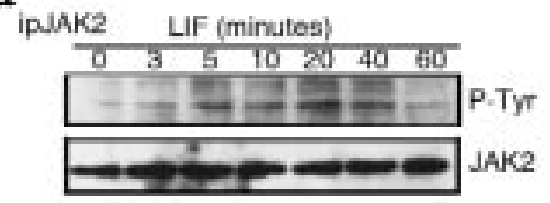

c

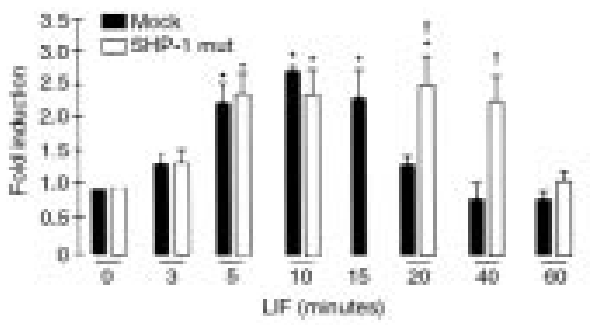

b
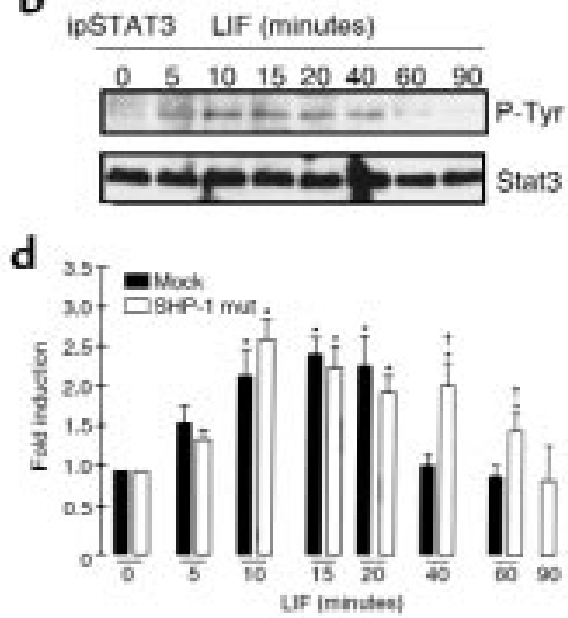

\section{Figure 4}

LIF-induced dephosphorylations of JAK2 and STAT3 are delayed in the mutant SHP-1-overexpressing transformants. Mutant SHP-1-overexpressing cells were treated for the indicated times with $1 \mathrm{nM}$ LIF. After cell lysis, immunoprecipitation and Western blots were performed as described in Figure 1, $a$ and $b$ ( $\mathbf{a}$ and $\mathbf{b}$, respectively). Quantification of kinetics of LIF-induced JAK2 (c) and STAT3 (d) tyrosine phosphorylation, observed in 3 different experiments from 3 independent clones of mock or mutant SHP-1-transfected cells, was performed. ${ }^{*} P$ $<0.05 \%$, LIF-treated versus control untreated cells. ${ }^{\dagger} P<0.05 \%$, SHP-1 mutant-transfected versus mock-transfected cells.

SHP-1 was LIF regulated, increased at 5 and 10 minutes of treatment, and decreased thereafter, concordant with STAT3 tyrosine phosphorylation after LIF stimulation. This result indicates that recruitment of STAT3 to the JAK2/SHP-1 complex is LIF dependent.

Kinetics of LIF-induced SHP-1 catalytic activity in AtT2O cells. To test whether SHP-1 catalytic properties were enhanced in LIF-stimulated AtT20 cells, we used an in vitro assay measuring dephosphorylation of an exogenous substrate, poly-Glu-Tyr, in the anti-SHP-1 immunoprecipitate. LIF-treated AtT20 cells were lysed; SHP-1 was immunoprecipitated with a monoclonal anti-SHP-1 antibody; and SHP-1 catalytic activity was measured in the immunoprecipitates (Figure 3). SHP1 activity obtained with an anti-mouse preimmune serum immunoprecipitate was subtracted from phosphatase activity values obtained with anti-SHP-1 immunoprecipitates to get specific SHP-1 activity. Increased SHP-1 tyrosine phosphatase activity was observed after 20 minutes of LIF treatment $(174 \% \pm 25$ of control; $P<0.05)$, peaked at 30 minutes $(245 \% \pm 27$ of control; $P<0.05$ ) and then decreased for up to 90 minutes. Thus, LIF-induced activation of SHP-1 might be responsible for JAK2 and STAT3 substrate dephosphorylation; indeed, the time course of LIF-induced SHP-1 catalytic activation correlated with that for LIFinduced JAK2 and STAT3 dephosphorylations.

SHP-1 catalytic inactive dominant negative mutant delays LIF-induced dephosphorylation of JAK2 and STAT3. To confirm the negative regulation of LIF signaling by SHP-1, we transfected AtT20 cells with cDNAs coding for either wild-type or a catalytically inactive form of SHP1. We initially confirmed the appropriate expression of both transformants in AtT20 cells and chose 3 clones with the highest levels of wild-type or mutant SHP-1.
Clones overexpressing the dominant negative form of SHP-1, or mock-transfected AtT20 cells, were used first to analyze the levels of JAK2 and STAT3 tyrosine phosphorylation after LIF treatment (Figure 4, a and b). Transformants were stimulated with $1 \mathrm{nM}$ LIF for up to 90 minutes and then lysed. Immunoprecipitations were performed with anti-JAK2 and anti-STAT3 antibodies, followed by antiphosphotyrosine antibody Western blotting analysis to observe levels of JAK2 and STAT3 tyrosine phosphorylation. Kinetics of LIFinduced JAK2 and STAT3 phosphorylations in mocktransfected AtT20 cells were similar to those in parental AtT20 cells, and quantifications obtained from 3 independent mock- and mutant SHP-1-transfected clones are represented in Figure 4, c and d. Whereas rapid dephosphorylations of JAK2 and STAT3 occurred in mock-transfected AtT20 cells as early as 20 minutes and 40 minutes, respectively (Figure 4, $\mathrm{c}$ and d), these dephosphorylations were significantly delayed in the mutant SHP-1 transformants cells until 60 minutes and 90 minutes, respectively (Figure 4, a-d). These results implied a role for SHP-1 in early tyrosine dephosphorylation of LIF-mediated JAK2 and STAT3 in AtT20 cells.

Role of SHP-1 in LIF-induced POMC transcriptional activation. Next, we investigated the functional importance of SHP-1 in LIF-mediated corticotroph action. We recently showed that STAT3 was a key signaling molecule for LIF induction of POMC transcription and ACTH secretion in AtT20 cells (8). Therefore, we reasoned that overexpression of wild-type or a mutated form of SHP-1 might influence the LIF-activation of this pathway. Three clones for mock-transfected AtT20 cells, wildtype, and mutated SHP-1 transformants were transiently transfected with POMC promoter-luciferase 
reporter construct and treated for 6 hours with LIF before cell lysis (Figure 5a). Results are expressed as foldinduction above control (i.e., LIF-stimulated cells versus unstimulated cells). LIF-induced POMC promoter activity decreased in wild-type SHP-1 transformants (3.4-fold $\pm 0.1 ; P<0.01)$ in comparison with mocktransfected cells (4.2-fold \pm 0.2$)$. In mutant SHP-1 transformants, luciferase was significantly enhanced (6.2fold $\pm 0.4 ; P<0.01)$ in comparison with its activity in mock-transfectant and wild-type SHP-1-overexpressing clones. To confirm the specificity of SHP-1 in LIF signaling, the cells were also treated with an analog of cyclic adenosine monophosphate (cAMP), dibutyryl cAMP (Figure 5b), which is known to stimulate POMC promoter activity in a cytokine-independent fashion (22). Luciferase gene activation was not different in mock SHP-1 (2.5-fold \pm 0.1 ), wild-type SHP-1 (2.4-fold \pm 0.1 ), or mutant SHP-1 (2.6-fold \pm 0.1$)$ transfected cells, indicating the specificity of SHP-1 in negatively regulating LIF signaling to POMC.

These results thus ascribe functional importance of SHP-1 association with the LIF-signaling molecules, JAK2 and STAT3, for POMC activation.

SOCS-3 is a component of LIF-induced dephosphorylation of JAK2 and STAT3. Next, we tested whether SOCS-3 might also be a component of early negative feedback of LIF signaling. We had previously shown that SOCS3 mRNA expression is LIF inducible, is maximal after 30 minutes, and is apparent for up to 8 hours of LIF stimulation (18). Therefore, we performed Western blotting to follow the kinetics of LIF-induced SOCS-3 expression at the protein level (Figure 6a). Immunoprecipitation of SOCS-3 with an anti-COOH-terminal region of SOCS-3 antibody, followed by Western blotting using an anti- $\mathrm{NH}_{2}$-terminal region of SOCS-3 antibody, showed that appearance of SOCS-3 protein is rapid, occurring within 20 minutes, is maximal after 40-60 minutes, and is no longer detectable after 90 minutes of LIF treatment. To investigate discrepancies between the time course for LIF-induced SOCS-3 mRNA and protein stability, an SOCS-3 Western blot was performed using a proteasome pathway inhibitor (LLnL) (Figure 6b). Pretreatment of AtT20 cells for 15 minutes with $10 \mu \mathrm{M}$ of LLnL (diluted in DMSO; final concentration of DMSO $=1 \%$ ) does not impact SOCS3 expression when these cells are not stimulated with 1 nM LIF (Figure 6b, lane 1). Pretreatment of AtT20 cells with $1 \%$ DMSO for 15 minutes does not impact either LIF-induced SOCS-3 expression or degradation: the same time course of LIF-induced SOCS-3 expression is observed in $0 \%$ DMSO (Figure 6a) or in 1\% DMSO (Figure 6b, lanes 2-4). However, pretreating AtT20 cells with $10 \mu \mathrm{M}$ LLnL for 15 minutes inhibited LIF-induced SOCS-3 degradation at 90 minutes, and SOCS-3 expression was still observed for up to 120 minutes and 180 minutes (Figure 6b, lanes 5-8).

SOCS-3 association with JAK2 was also assessed in an anti-JAK2 Western blot using anti-SOCS-3 immunoprecipitate (Figure 6c). After 60 minutes of LIF treat- ment, a maximal association between JAK2 and SOCS3 was evident and decreased very quickly. This result was confirmed in an anti-SOCS-3 Western blot using anti-JAK2 immunoprecipitate: maximal coimmunoprecipitation between JAK2 and SOCS-3 was also observed at 60 minutes (data not shown). To ascribe a functional importance for SOCS-3 binding to JAK2, we investigated whether SOCS-3 directly inhibited JAK2 autophosphorylation. Using SOCS-3-transfected and mock-transfected AtT20 cells (18), we performed an antiphosphotyrosine Western blot experiment using anti-JAK2 immunoprecipitates (Figure 6d). LIFinduced phosphotyrosine levels of JAK2 in mock-transfected cells (Figure 6d, lanes 4-6) were greater than in SOCS-3-overexpressing cells (Figure 6d, lanes 1-3). This result confirms that blockade of LIF signaling in SOCS-3-overexpressing cells results directly from SOCS-3-dependent inhibition of JAK2 autophosphorylation and activation.

Definition of SOCS-3 functional domains. We then investigated which domains of SOCS-3 were responsible for its function by constructing SOCS-3 fusion proteins (Figure 7a). Correct expression of each construct, transiently transfected in AtT20 cells, was confirmed in an anti-SOCS-3 Western blot using anti-X-press immunoprecipitates (data not shown). This experiment indeed showed similar protein expression levels
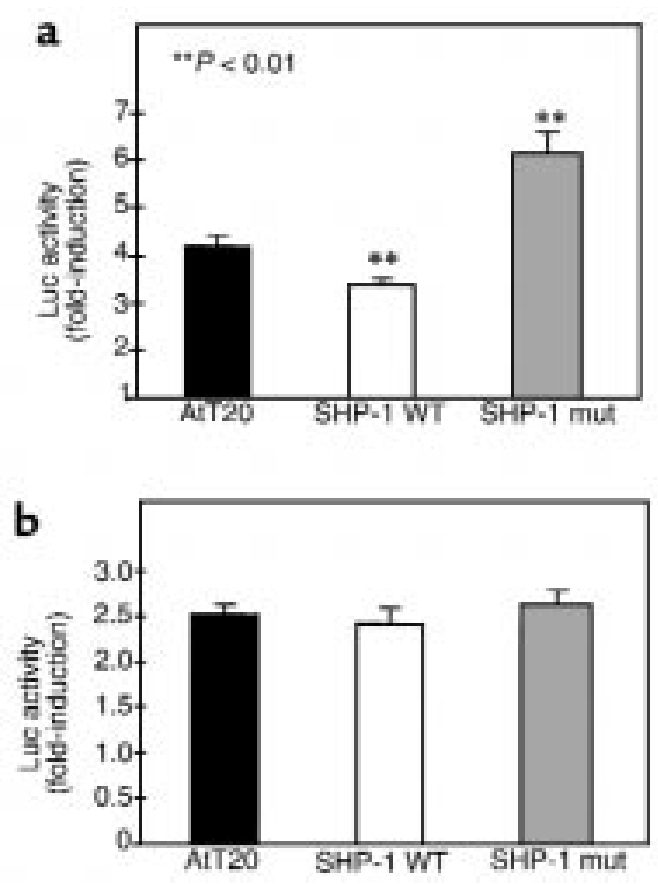

Figure 5

SHP-1 catalytic activity influences LIF-induced POMC promoter activity. Mock-, wild-type (WT) SHP-1-, or mutant (mut) SHP1 -transfected AtT20 cells were transiently transfected with rat POMC promoter fused to the luciferase reporter. Cells were then treated for 6 hours with $1 \mathrm{nM}$ LIF (a) or $5 \mathrm{mM}$ dibutyryl cAMP (b). Luciferase activity was measured as described in Methods. The results are representative of 3 separate experiments using 3 independent clones and are expressed as fold-induction above control. 

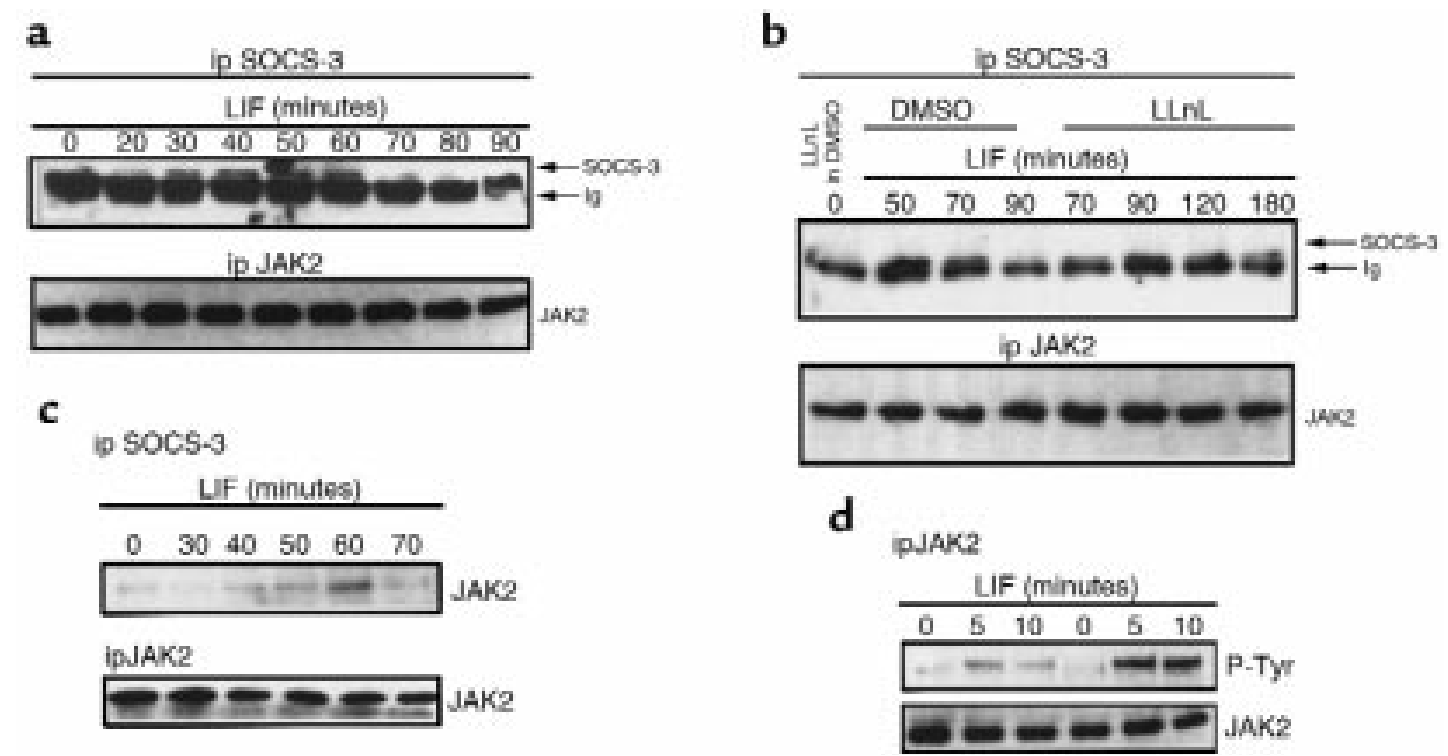

\begin{abstract}
Figure 6
SOCS-3 protein is LIF-inducible, binds in vivo to JAK2, and blocks LIF-induced JAK2 tyrosine phosphorylation. (a) AtT20 cells were treated for the indicated times with $1 \mathrm{nM}$ LIF. After cell lysis, half the lysates were used in immunoprecipitations using a polyclonal antibody recognizing $\mathrm{COOH}$-terminal of SOCS-3; the immunoprecipitates were separated on a $15 \%$ SDS-polyacrylamide gel and then immunoblotted with a polyclonal antibody recognizing $\mathrm{NH}_{2}$-terminal of SOCS-3. To verify equal loading of proteins, the other half of the lysates were used in immunoprecipitations using a polyclonal anti-JAK2 antibody, run on a 7.5\% SDS-polyacrylamide gel and blotted with an anti-JAK2 antibody. (b) AtT20 cells were pretreated with 1\% DMSO (vehicle) (lanes 2-4) or with $10 \mu \mathrm{M} \mathrm{LLnL}$ (dissolved in DMSO) for 15 minutes (lanes 1, 5-8), and then treated with $1 \mathrm{nM} \mathrm{LIF}$ for the indicated time (lanes 2-8). The experiment was also performed as described in a. (c) AtT20 cells were treated for the indicated times with $1 \mathrm{nM}$ LIF. After cell lysis, half of the lysates were used in immunoprecipitations using a polyclonal antibody recognizing $\mathrm{COOH}$-terminal of SOCS-3; the immunoprecipitates were separated on a $7.5 \%$ SDS-polyacrylamide gel and then immunoblotted with a polyclonal anti-JAK2 antibody. To verify equal loading of proteins, the other half of the lysates were used in immunoprecipitations using a polyclonal anti-JAK2 antibody, run on a 7.5\% SDS-polyacrylamide gel, and blotted with an anti-JAK2 antibody. (d) Treatment of mock-transfected AtT20 cells (lanes 4-6) or SOCS-3-overexpressing cells (lanes 1-3) were performed as indicated. Immunoprecipitation was then performed using an anti-JAK2 antibody. The immune complexes were run on a 7.5\% SDS-polyacrylamide gel, and a Western blot was performed using an antiphosphotyrosine antibody. To verify equal loading of proteins, the membrane was stripped and subjected to a Western blot using an anti-JAK2 antibody.
\end{abstract}

for all SOCS-3 constructs. The fusion protein 1-226, corresponding to the full SOCS-3 sequence, and the fusion protein 19-226, corresponding to the pre-SH2, $\mathrm{SH} 2$, post-SH2, and SOCS box regions of SOCS-3 (Figure 7a), efficiently inhibited LIF-induced POMC promoter activity $(100 \%$ and $99.8 \% \pm 4.5$ of POMC luciferase activity, respectively, versus $153 \% \pm 8$ for the empty plasmid; $P \leq 0.001)$. By opposition, the fusion proteins 19-127, 19-186, or 45-226 - corresponding, respectively, to the pre-SH2 and $\mathrm{SH} 2$ domains; to the pre-SH2, SH2, and post-SH2 domains; or to the SH2, post-SH2, and SOCS box domains - were inactive (Figure $7 \mathrm{~b}$ ). Thus, this result confirms the critical role of the pre-SH2 and SH2 domains of SOCS-3, as also described for SSI-1/SOCS-1, but also ascribes a critical role for the SOCS box for inhibition of LIF-induced POMC promoter activity (32).

\section{Discussion}

We show here that the time course of pituicyte LIF stimulation is associated with tyrosine phosphorylation of JAK2 and STAT3, 2 well-recognized substrates for LIF in these cells $(8,21)$. Earlier effects of LIF on JAK2 and STAT3 phosphorylation occurred after 5 minutes of stimulation with JAK2 and STAT3, whereas these phosphorylated substrates were undetectable after 20 or 40 minutes, respectively (Figure 1, a and b). The apparent failure to detect these phosphorylated molecules is probably not due to their degradation because JAK2 and STAT3 protein levels are sustained during the time course of LIF stimulation, suggesting rapid dephosphorylation of both of these LIF substrates.

We investigated the respective roles of SHP-1 and SOCS-3 in downregulating LIF signaling to POMC. Tyrosine phosphatases act both positively and negatively in cytokine and growth factor signal transduction (23). Although the SH2 domain-containing phosphatase SHP-1 has been implicated in positively mediating EGF- and IFN- $\gamma$-stimulated STAT transcription factor activation in HeLa cells (10), this phosphatase has mainly been described as a negative regulator of cell signaling. SHP-1 controls erythropoietin signal transduction by regulating receptor-associated JAK2 tyrosine phosphorylation (14), and it controls JAK1 tyrosyl phosphorylation in response to IFN- $\alpha / \beta$ (24). SHP-1 is also a negative regulator of liver GH signaling by regulating the rates of JAK2 and STAT protein inactivation $(11,12)$. These results support the 
concept that inactivation of receptor-associated JAK may be a general mechanism by which SHP-1 regulates multiple cytokine receptor signaling pathways.

We show SHP-1 to be associated with the LIF-stimulated JAK2-STAT3 complex. Whereas the association between SHP-1 and JAK2 is apparently independent of LIF stimulation, interaction between SHP-1 and STAT3 is LIF regulated. LIF induces STAT3 recruitment onto the phosphatase with a time course that parallels that of induction of STAT3 phosphorylation. JAK2 is a constitutive partner of gp130 (6), and LIF induces dimerization of the cytoplasmic domains of LIFR $\alpha$ and gp130 subunits, which, in turn, activates JAK2 by autophosphorylation (6). Our results also demonstrate a constitutive association between SHP-1 and JAK2, independent of JAK2 phosphorylation. Such an association of SHP-1 with nonphosphorylated receptor has been previously reported for the IFN- $\alpha / \beta$ receptor $\alpha$ subunit (24) and for the erythropoietin receptor (14). Furthermore, SHP-1 has been reported to constitutively interact with Tyk2 (25) or to be associated with JAK2 through SH2-phosphotyrosine-independent interactions $(13,26)$. We show that LIF induces SHP-1 activation and subsequent dephosphorylation of JAK2. Furthermore, we demonstrated that the association between SHP-1 and STAT3 was LIF regulated and paralleled kinetics for LIF-induced tyrosine phosphorylation of STAT3. Although STAT3 interaction with SHP-1 has not been thus far reported, activated STAT5b can bind directly to SHP-1 in the nucleus (11), thus STAT3 might bind directly or indirectly through gp130 to SHP-1. We also showed that the LIFinduced SHP-1 catalytic activation is time correlated with JAK2 and STAT3 dephosphorylation.

The SHP-1 relevance in LIF regulation of corticotroph function was studied in mutant SHP-1-overexpressing cells, which showed delayed JAK2 and STAT3 dephosphorylations until after 60 minutes of LIF stimulation, in comparison to mock-transfected cells. We also showed that LIF induction of POMC promoter activity was reduced in wild-type SHP1 -overexpressing clones and enhanced in mutant SHP1 -overexpressing transformants. SHP-1 specificity in LIF signaling was demonstrated because no difference of POMC promoter activity was observed after stimulation of transformants with dibutyryl cAMP, which is known to stimulate POMC promoter activity through an LIF-independent pathway (22).

On the basis of our observations, we propose a model (Figure 8) by which SHP-1 is constitutively associated with JAK2 in a tyrosine phosphorylation-independent manner. After LIF stimulation, JAK2 is activated. JAK2 tyrosine phosphorylates itself, as well as gp130 and STAT3. These new phosphotyrosine sites might be potential docking sites for the SHP-1 SH2 domains, resulting in SHP-1 activation. Indeed, enzymatic activation of SHP-1 has been reported to occur through association of its $\mathrm{SH} 2 \mathrm{COOH}$ - or $\mathrm{NH}_{2}$-terminal domains with phosphotyrosine docking sites of hematopoietic growth factor receptors (14, 27-29). Increased tyrosine phosphatase activity of SHP-1 results in dephosphorylation of JAK2 (14), therefore inhibiting its kinase activity and resulting in dephosphorylation of its substrates, namely, gp130 and STAT3. Thus, these results identify SHP-1 as an early negative feedback regulator of LIF signaling in AtT20 cells.

SOCS proteins inhibit cytokine-induced signal transduction in many systems (16-18) and bind in vitro to JAKs (30). We previously reported that LIF rapidly activates SOCS-3 mRNA expression within 30 minutes. SOCS-3 overexpression in these cells inhibits LIFinduced gp130 and STAT3 tyrosine phosphorylation and blocks LIF-induced POMC promoter activity (18). We also show here that LIF induces endogenous SOCS3 protein's appearance and binding to JAK2 with a time course correlating with JAK2 dephosphorylation, indicating that SOCS-3 blocks LIF signaling by binding and inhibiting JAK2 activity. This is confirmed in SOCS-3-overexpressing cells that show reduced LIFinduced JAK2 phosphorylation. Maximal in vivo association between SOCS-3 and JAK2 occurs at $60 \mathrm{~min}-$ utes, suggesting that SOCS-3-induced inactivation of JAK2 is slightly delayed, compared with SHP-1 action. This result may also explain JAK2 and STAT3 dephosphorylations occurring even in mutant SHP-1-overexpressing transformants after 60 minutes.

a
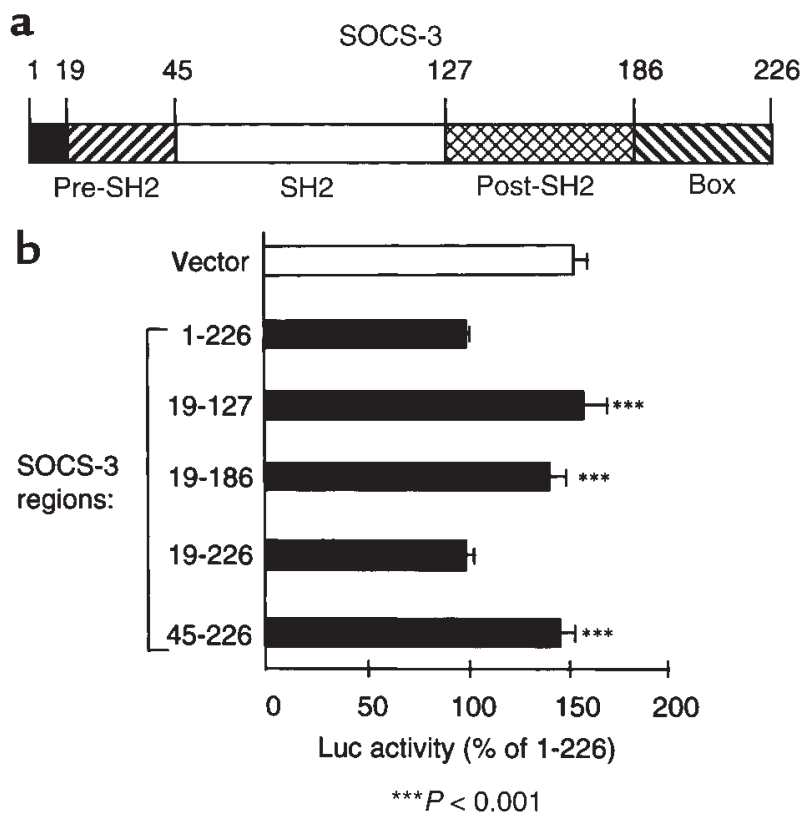

\section{Figure 7}

Definition of the functional domains of SOCS-3. (a) Description of the different domains of SOCS-3. (b) Fusion protein corresponding to a $\mathrm{NH}_{2}$-terminal tag from the pcDNA3.1/His vector (Invitrogen) fused to different portions of SOCS-3. These constructs were transiently transfected with rat POMC promoter fused to the luciferase reporter in AtT20 cells. Cells were then treated for 6 hours with $1 \mathrm{nM}$ LIF. Luciferase activity was measured as described in Methods. The results are representative of 3 separate experiments and are expressed as percentage of control $(100 \%=$ full-length SOCS-3 $)$. 


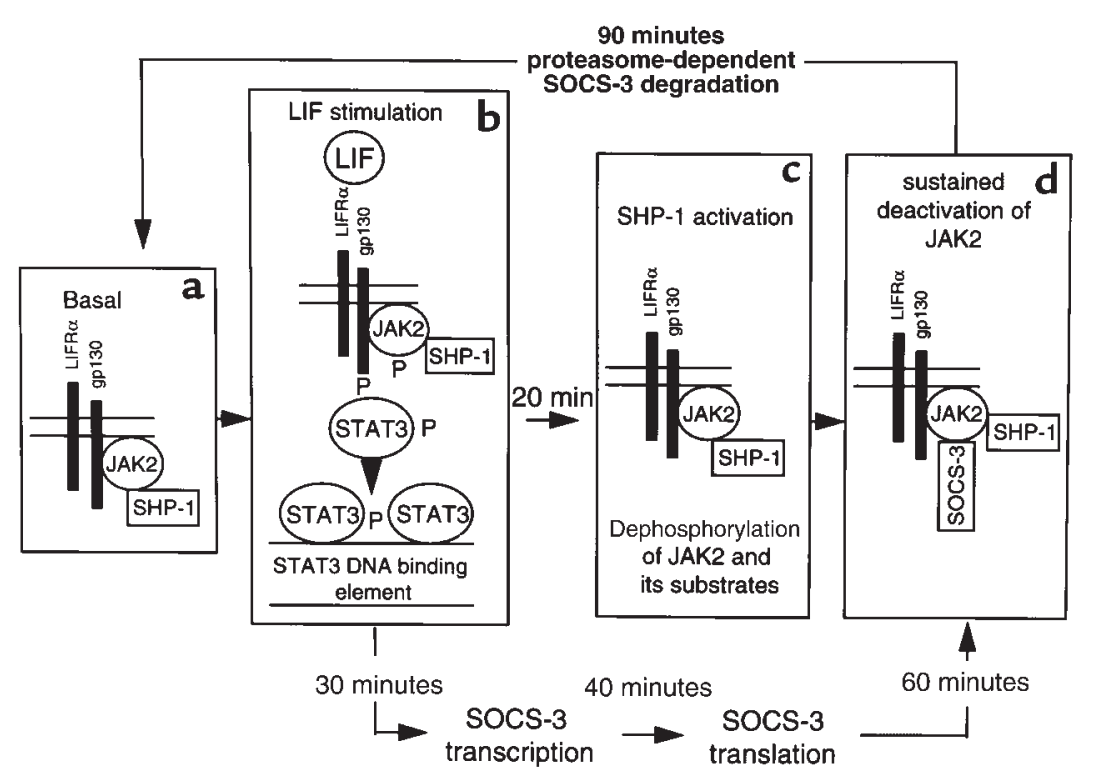

\section{Figure 8}

Model for SHP-1 and SOCS-3 regulation of LIF transduction signal. (a) SHP-1 is constitutively associated with JAK2 in a tyrosine phosphorylation-independent manner. (b) After LIF stimulation, heterodimerization occurs between LIFR $\alpha$ and gp130 subunits, which activates JAK2 activity. JAK2 tyrosine phosphorylates itself as well as gp130, which allows recruitment of STAT3 through its SH2 domain onto gP130 docking sites. STAT3 is also tyrosine-phosphorylated by JAK2 and therefore activated. STAT3 then homodimerizes or heterodimerizes with STAT1, which accounts for its nuclear translocation. (c) SHP-1 might be activated because its partners (JAK2 and STAT3) become tyrosine-phosphorylated. Increased tyrosine phosphatase activity of SHP-1 directly dephosphorylates JAK2, therefore inhibiting its kinase activity and resulting in dephosphorylation of its substrates. Once gp130, JAK2, and STAT3 are dephosphorylated, STAT3 leaves the receptor complex, which also returns to the basal state. Activation of SHP-1 is maximal at 30 minutes of LIF stimulation. (d) Sustained deactivation of LIF signaling also occurs through LIF-induced SOCS-3 protein synthesis and binding to JAK2, which appears maximally between 40 and 60 minutes of LIF stimulation. SOCS-3 inhibits JAK2 kinase activity and is then degraded after 90 minutes.

To elucidate variations in stability of SOCS-3 protein (<90 minutes) and SOCS-3 mRNA (> 8 hours) after LIF treatment, we used a proteasome pathway inhibitor. Indeed, CIS has previously been shown to be sensitive to degradation regulated by the proteasome (31). Furthermore, SOCS-1 deletion constructs lacking the "SOCS-box" were expressed in mammalian cells only in the presence of proteasome inhibitors (32). Blocking the proteasome pathway prolongs SOCS-3 protein expression until 3 hours after LIF treatment, demonstrating a role for the proteasome for LIFinduced SOCS-3 degradation.

Using fusion proteins containing different segments of SOCS-3 protein, we show here that amino acids sequences 19-226 are critical for SOCS-3-mediated inhibition of LIF-induced POMC promoter activity. Recent reports, in which deletions of the SSI-1/SOCS1 sequence were tested on IL-6-mediated stimulation of the IRF-1 promoter (32) and on M1 cell differentiation (33), showed that the pre-SH2 domain and the SH2 domain contribute to SSI-1/SOCS-1 function and that the SOCS box is required to protect SSI-1 from proteolytic degradation (32). However, in 1 report, SOCS-1 was deleted from its SOCS box and still inhibited JAK1 kinase activity to the same extent as the fulllength SOCS-1 (33). However, we show here that the sequences $\mathrm{COOH}$-terminal to the $\mathrm{SH} 2$ domain of SOCS-3 are also critical for in vivo SOCS-3 function. As suggested elsewhere, sequences $\mathrm{COOH}$-terminal of the SH2 domain, and particularly the SOCS box, might be required for SOCS-3 stability and prevention of proteasome-induced degradation $(32,34)$. Increased stability of SOCS-1 protein has been shown to be mediated through interaction between the SOCS box and elongin BC (34). However, we show here the degradation of wild-type SOCS-3, which also contains the SOCS box, to occur in vivo after approximately $90 \mathrm{~min}-$ utes of LIF treatment. This also suggests either that the multicomplex SOCS-elongin BC is not fully protective against SOCS-3 degradation or that a LIF-inducible event targets SOCS-3 for degradation at this time point of LIF treatment. Binding between SOCS-3 and JAK2 might indeed constitute 1 of these events. SOCS3/JAK2 association may lead either to a special folding of SOCS-3, which masks the protective SOCS box, or to the dissociation between the SOCS box and elongin BC. Alternatively, SOCS/JAK2 association may result in SOCS-3 tyrosine phosphorylation. Indeed, IL2 -induced tyrosine phosphorylation of SOCS-3 was recently demonstrated (35), and phosphorylationdependent degradation by the proteasome pathway has been reported for STAT1 (36).

In conclusion, we report that both SHP-1 and SOCS3 negatively regulate LIF corticotroph function in AtT20 cells. The mechanisms for JAK2 inactivation differ for the 2 proteins. SHP- 1 acts by tyrosine-dephos- 
phorylating its substrates and thus affecting the balance of phosphorylated versus dephosphorylated protein, to prevent excess effects of ligand. SOCS-3 might inhibit JAK2 by directly interacting with its JH1 domain, essential for its kinase function. Both mechanisms might be complementary to inhibit LIF corticotroph function; whereas SHP- 1 is constitutively present and rapidly activated by LIF, SOCS-3 is absent at the basal level and its expression is LIF-inducible.

Cytokine signal transduction thus involves redundancy of JAK/STAT interactions, phosphatase function, and SOCS protein autoregulation. These results demonstrate a complex mechanism for the plastic "onoff" cytokine-mediated regulation of the HPA axis at the immunoneuroendocrine interface.

\section{Acknowledgments}

We are grateful to C. Nahmias (Institut Cochin de Génétique Moléculaire, CNRS UPR 0415, France) for providing the plasmid encoding the SHP-1 mutant. We thank C.J. Auernhammer for providing AtT20 cells transfected with SOCS-3, and M.C. Zatelli and P. A. Merkel for their critical reading of the manuscript. This study was supported by a grant from the National Institutes of Health (R01-DK50238) and by the Doris Factor Molecular Endocrinology Laboratory. C. Bousquet is a recipient of a doctoral fellowship from the Ministère de l'Education Nationale, de l'Enseignement Supérieur et de la Recherche, France.

1. Hibi, M., Nakajima, K., and Hirano, T. 1996. IL-6 cytokine family and signal transduction: a model of the cytokine system. J. Mol. Med. 74:1-12.

2. Chesnokova, V., Auernhammer, C.J., and Melmed, S. 1998. Murine LIF gene disruption attenuates the hypothalamo-pituitary-adrenal axis stress response. Endocrinology. 139:2209-2216.

3. Akita, S., et al. 1995. Human and murine pituitary expression of LIF: novel intrapituitary regulation of adrenocoeticotropin synthesis and secretion. J. Clin. Invest. 95:1288-1298.

4. Wang, Z., Ren, S.G., and Melmed, S. 1996. Hypothalamic and pituitary LIF gene expression in-vivo: a novel endotoxin-inducible neuroendocrine interface. Endocrinology. 137:2947-2953.

5. Bousquet, C., Ray, D.W., and Melmed, S. 1997. A common proopiomelanocortin element mediates LIF and CRH transcriptional synergy. J. Biol. Chem. 272:10551-10557.

6. Ihle, J.N. 1995. The janus protein tyrosine kinase family and its role in cytokine signaling. Adv. Immunol. 60:1-35.

7. Horvath, C.M., and Darnell, J.E., Jr. 1997. The state of the STATs: recent developments in the study of signal transduction to the nucleus. Curr. Opin. Cell Biol. 9:233-239.

8. Bousquet, C., and Melmed, S. 1999. Critical role for STAT3 in murine pituitary ACTH leukemia inhibitory factor (LIF) signaling. J. Biol. Chem. 274:10723-10730.

9. Fauman, E.B., and Saper, M.A. 1996. Structure and function of the protein tyrosine phosphatases. Trends Biochem. Sci. 21:413-417.

10. You, M., and Zhao, Z. 1997. Positive effects of SH2 domain-containing tyrosine phosphatase SHP-1 on epidermal growth factor- and interferon- $\boldsymbol{\gamma}$-stimulated activation of STAT transcription factors in HeLa cells. J. Biol. Chem. 272:23376-23381.

11. Ram, P.A., and Waxman, D.J. 1997. Interaction of growth hormone-activated STATs with SH2-containing phosphotyrosine phosphatase SHP1 and nuclear JAK2 tyrosine kinase. J. Biol. Chem. 272:17694-17702.

12. Hackett, R.H., et al. 1997. Mapping of a cytoplasmic domain of the human growth hormone receptor that regulates rates of inactivation of
JAK2 and STAT proteins. J. Biol. Chem. 272:11128-11132.

13. Jiao, H., et al. 1996. Direct association with and dephosphorylation of Jak2 kinase by the $\mathrm{SH} 2$-domain containing protein tyrosine phosphatase SHP-1. Mol. Cell. Biol. 16:6985-6992.

14. Klingmuller, U., Lorenz, U., Cantley, L.C., Neel, B.G., and Lodish, H.F. 1995. Specific recruitment of SH-PTP1 to the erythropoietin receptor causes inactivation of JAK2 and termination of proliferative signals. Cell. 80:729-738

15. Hilton, D.J., Richardson, R.T., Alexander, W.S., Viney, E.M., Willson, T.A., et al. 1998. Twenty proteins containing a C-terminal SOCS box form five structural classes. Proc. Natl. Acad. Sci. USA. 95:114-119.

16. Adams, J.E., et al. 1998. Growth hormone preferentially induces the rapid, transient expression of SOCS-3, a novel inhibitor of cytokine receptor signaling. J. Biol. Chem. 273:1285-1287.

17. Bjorbaek, C., Elmquist, J.K., Frantz, J.D., Shoelson, S.E., and Flier J.S. 1998. Identification of SOCS-3 as a potential mediator of central leptin resistance. Mol. Cell. 1:619-625.

18. Auernhammer, C.J., Chesnokova, V., Bousquet, C., and Melmed, S. 1998. Pituitary corticotroph SOCS-3: novel intracellular regulation of cytokine-mediated proopiomelanocortin gene expression and ACTH secretion. Mol. Endocrinol. 12:954-961.

19. Auernhammer, C.J., Bousquet, C., and Melmed, S. 1999. Autoregulation of pituitary corticotroph SOCS-3 expression: characterization of the murine SOCS-3 promoter. Proc. Natl. Acad. Sci. USA. 96:6964-6969.

20. Bousquet, C., et al. 1998. sst2 somatostatin receptor mediates negative regulation of insulin receptor signaling through the tyrosine phosphatase SHP-1. J. Biol. Chem. 273:7099-7106

21. Ray, D.W., Ren, S.G., and Melmed, S. 1996. Leukemia inhibitory factor (LIF) stimulates proopiomelanocortin (POMC) expression in a corticotroph cell line. Role of STAT pathway. J. Clin. Invest. 97:1852-1859.

22. Boutillier, A.L., Gaiddon, C., Lorang, D., Roberts, J.L., and Loeffler, J.P. 1998. Transcriptional activation of the proopiomelanocortin gene by cyclic AMP-responsive element binding protein. Pituitary. 1:33-43.

23. Feng, G.S., and Pawson, T. 1994. Phosphotyrosine phosphatases with SH2 domains: regulators of signal transduction. Trends Genet. 10:54-58.

24. David, M., Chen, H.E., Goelz, S., Larner, A.C., and Nell, B.G. 1995. Differential regulation of the alpha/beta interferon-stimulated Jak-Stat pathway by the SH2 domain-containing tyrosine phosphatase SHPTP1. Mol. Cell. Biol. 15:7050-7058.

25. Yetter, A., et al. 1995. Association of the interferon-dependent tyrosine kinase Tyk-2 with the hematopoietic cell phosphatase. J. Biol. Chem. 270:18179-18182.

26. Damen, J.E., et al. 1995. Tyrosine 343 in the erythropoietin receptor positively regulates erythropoietin-induced cell proliferation and Stat 5 activation. EMBO J. 14:5557-5568.

27. D'Ambrosio, D., et al. 1995. Recruitment and activation of PTP1C in negative regulation of antigen receptor signaling by Fc gamma RIIB1. Science. 268:293-297.

28. Pei, D., Lorenz, U., Klingmuller, U., Neel, B.G., and Walsh, C.T. 1994. Intramolecular regulation of protein tyrosine phosphatase SH-PTP1: a new function for Src homology 2 domains. Biochemistry. 33:15483-15493.

29. Yi, T., Zhang, J., Miura, O., and Ihle, J.N. 1995. Hematopoietic cell phosphatase associates with erythropoietin (Epo) receptor after Epo-induced receptor tyrosine phosphorylation: identification of potential binding sites. Blood. 85:87-95.

30. Endo, T.A., et al. 1997. A new protein containing an SH2 domain that inhibits JAK kinases. Nature. 387:921-924.

31. Yoshimura, A., et al. 1995. A novel cytokine-inducible gene CIS encodes an $\mathrm{SH} 2$-containing protein that binds to tyrosine-phosphorylated interleukin 3 and erythropoietin receptors. EMBO J. 14:2816-2826.

32. Narazaki, M., et al. 1998. Three distinct domains of SSI-1/SOCS-1/JAB protein are required for its suppression of interleukin 6 signaling. Proc. Natl. Acad. Sci. USA. 95:13130-13134.

33. Nicholson, S.E., et al. 1999. Mutational analyses of the SOCS proteins suggest a dual domain requirement but distinct mechanisms for inhibition of LIF and IL-6 signal transduction. EMBO J. 18:375-385.

34. Kamura, T., et al. 1998. The elongin BC complex interacts with the conserved SOCS-box motif present in members of the SOCS, ras, WD-40 repeat, and ankyrin repeat families. Genes Dev. 12:3872-3881.

35. Cohney, S.J., et al. 1999. SOCS-3 is tyrosine phosporylated in response to interleukin-2 and suppresses STAT5 phosphorylation and lymphocyte proliferation. Mol. Cell. Biol. 19:4980-4988.

36. Kim, T.K., and Maniatis, T. 1996. Regulation of interferon-gamma-activated STAT1 by the ubiquitin-proteasome pathway. Science. 273:1717-1719. 